\title{
Predictability of band-limited, high-frequency, and mixed processes in the presence of ideal low-pass filters
}

\author{
Nikolai Dokuchaev \\ Department of Mathematics, Trent University, Ontario, Canada
}

\begin{abstract}
Pathwise predictability of continuous time processes is studied in deterministic setting. We discuss uniform prediction in some weak sense with respect to certain classes of inputs. More precisely, we study possibility of approximation of convolution integrals over future time by integrals over past time. We found that all band-limited processes are predictable in this sense, as well as high-frequency processes with zero energy at low frequencies. It follows that a process of mixed type still can be predicted if an ideal low-pass filter exists for this process.
\end{abstract}

Key words: prediction, ideal low-pass filters, band-limited processes, Hardy spaces, causal estimators.

AMS 2000 classification : 60G25, 93E10, 42B30.

PACS 2008 numbers: 02.30.Mv, 02.30.Nw, 02.30.Yy, 07.05.Mh, 07.05.Kf

\section{Introduction}

We study pathwise predictability of continuous time processes in deterministic setting. It is well known that certain restrictions on frequency distribution can ensure additional opportunities for prediction and interpolation of the processes. The classical result is Nyquist-Shannon-Kotelnikov interpolation theorem for the band-limited processes. There are related predictability results for these processes (see, e.g., Wainstein and Zubakov (1962), Beutler (1966), Brown(1969), Slepian (1978), Knab (1981), Papoulis (1985), Marvasti (1986), Vaidyanathan (1987), Lyman et al (2000, 2001)).

In the present paper, we study a special kind of weak predictability such that convolution integrals over future can be approximated by convolution integrals over past times representing historical observations. We found some cases when this approximation can be made uniformly over a wide class of input processes. We found that all band-limited 
processes are predictable in this sense. Similar result is obtained for high-frequency processes. For the processes of mixed type, we found that the similar predictability can be achieved when the model allows a low pass filter that acts as an ideal low-pass filter for this process. These results can be a useful addition to the existing theory of band-limited processes. The novelty is that we consider predictability of both high frequent and bandlimited processes in a weak sense uniformly over classes of input processes. In addition, we suggest a new type of predictor. Its kernel is given explicitly in the frequency domain.

\section{Definitions}

Let II denote the indicator function, $\mathbf{R}^{+} \triangleq[0,+\infty), \mathbf{C}^{+} \triangleq\{z \in \mathbf{C}: \operatorname{Re} z>0\}, i=\sqrt{-1}$.

For complex valued functions $x \in L_{1}(\mathbf{R})$ or $x \in L_{2}(\mathbf{R})$, we denote by $X=\mathcal{F} x$ the function defined on $i \mathbf{R}$ as the Fourier transform of $x$;

$$
(\mathcal{F} x)(i \omega)=\int_{-\infty}^{\infty} e^{-i \omega t} x(t) d t, \quad \omega \in \mathbf{R} .
$$

If $x \in L_{2}(\mathbf{R})$, then $X$ is defined as an element of $L_{2}(\mathbf{R})$ (meaning $L_{2}(i \mathbf{R})$ ).

For $v(\cdot) \in L_{2}(\mathbf{R})$ such that $v(t)=0$ for $t<0$, we denote by $\mathcal{L} v$ the Laplace transform

$$
V(p)=(\mathcal{L} v)(p) \triangleq \int_{0}^{\infty} e^{-p t} v(t) d t, \quad p \in \mathbf{C}^{+} .
$$

Let $H^{r}$ be the Hardy space of holomorphic on $\mathbf{C}^{+}$functions $h(p)$ with finite norm $\|h\|_{H^{r}}=\sup _{s>0}\|h(s+i \omega)\|_{L_{r}(\mathbf{R})}, r \in[1,+\infty]$ (see, e.g., Duren (1970)).

Let $\Omega>0$ be given.

Definition 1 Let $\mathcal{K}$ be the class of functions $k: \mathbf{R} \rightarrow \mathbf{R}$ such that $k(t)=0$ for $t>0$ and such that $K=\mathcal{F} k$ is

$$
K(i \omega)=\frac{d(i \omega)}{\delta(i \omega)}
$$

where $d(\cdot)$ and $\delta(\cdot)$ are polynomials such that $\operatorname{deg} d<\operatorname{deg} \delta$, and if $\delta(p)=0$ for $p \in \mathbf{C}$ then $\operatorname{Re} p>0,|\operatorname{Im} p|<\Omega$.

Note that the class $\mathcal{K}$ is quite wide: it consists of linear combinations of functions $q(t) e^{\lambda t} \mathbb{I}_{\{t \leq 0\}}$, where $\lambda \in \mathbf{C}, \operatorname{Re} \lambda>0,|\operatorname{Im} \lambda|<\Omega$, and where $q(t)$ is a polynomial.

Definition 2 Let $\widehat{\mathcal{K}}$ be the class of functions $\widehat{k}: \mathbf{R} \rightarrow \mathbf{R}$ such that $k(t)=0$ for $t<0$ and such that $K(\cdot)=\mathcal{L} \widehat{k} \in H^{2} \cap H^{\infty}$.

We are going to study linear predictors in the form $\widehat{y}(t)=\int_{-\infty}^{t} \widehat{k}(t-s) x(s) d s$ for the processes $y(t)=\int_{t}^{+\infty} k(t-s) x(s) d s$, where $k \in \mathcal{K}$ and $\widehat{k} \in \widehat{K}$. The predictors use historical values of currently observable process $x(\cdot)$. 
Definition 3 Let $\mathcal{X}=\{x(\cdot)\}$ be a class of functions $x: \mathbf{R} \rightarrow \mathbf{C}$. Let $r \in[1,+\infty]$.

(i) We say that the class $\mathcal{X}$ is $L_{r}$-predictable in the weak sense if, for any $k(\cdot) \in \mathcal{K}$, there exists a sequence $\left\{\widehat{k}_{m}(\cdot)\right\}_{m=1}^{+\infty}=\left\{\widehat{k}_{m}(\cdot, \mathcal{X}, k)\right\}_{m=1}^{+\infty} \subset \widehat{\mathcal{K}}$ such that

$$
\left\|y-\widehat{y}_{m}\right\|_{L_{r}(\mathbf{R})} \rightarrow 0 \quad \text { as } \quad m \rightarrow+\infty \quad \forall x \in \mathcal{X},
$$

where

$$
y(t) \triangleq \int_{t}^{+\infty} k(t-s) x(s) d s, \quad \widehat{y}_{m}(t) \triangleq \int_{-\infty}^{t} \widehat{k}_{m}(t-s) x(s) d s .
$$

(ii) Let the set $\mathcal{F}(\mathcal{X}) \triangleq\{X=\mathcal{F} x, \quad x \in \mathcal{X}\}$ be provided with a norm $\|\cdot\|$. We say that the class $\mathcal{X}$ is $L_{r}$-predictable in the weak sense uniformly with respect to the norm $\|\cdot\|$, if, for any $k(\cdot) \in \mathcal{K}$ and $\varepsilon>0$, there exists $\widehat{k}(\cdot)=\widehat{k}(\cdot, \mathcal{X}, k,\|\cdot\|, \varepsilon) \in \widehat{\mathcal{K}}$ such that

$$
\|y-\widehat{y}\|_{L_{r}(\mathbf{R})} \leq \varepsilon\|X\| \quad \forall x \in \mathcal{X}, \quad X=\mathcal{F} x .
$$

Here $y(\cdot)$ is the same as above, $\widehat{y}(t) \triangleq \int_{-\infty}^{t} \widehat{k}(t-s) x(s) d s$.

We call functions $\widehat{k}(\cdot)$ in Definition 3 predictors or predicting kernels.

\section{The main result}

Let $\Omega>0$ be the same as in the definition of $\mathcal{K}$, and let

$$
\begin{aligned}
& \mathcal{X}_{L} \triangleq\left\{x(\cdot) \in L_{2}(\mathbf{R}): X(\omega)=0 \quad \text { if } \quad|\omega|>\Omega, \quad X=\mathcal{F} x\right\}, \\
& \mathcal{X}_{H} \triangleq\left\{x(\cdot) \in L_{2}(\mathbf{R}): X(\omega)=0 \quad \text { if } \quad|\omega|<\Omega, \quad X=\mathcal{F} x\right\} .
\end{aligned}
$$

In particular, $\mathcal{X}_{L}$ is a class of band-limited processes, and $\mathcal{X}_{H}$ is a class of high-frequency processes.

\subsection{Predictability of band-limited and high-frequency processes from $L_{2}$}

Theorem 1 (i) The classes $\mathcal{X}_{L}$ and $\mathcal{X}_{H}$ are $L_{2}$-predictable in the weak sense.

(ii) The classes $\mathcal{X}_{L}$ and $\mathcal{X}_{H}$ are $L_{\infty}$-predictable in the weak sense uniformly with respect to the norm $\|\cdot\|_{L_{2}(\mathbf{R})}$.

(iii) For any $q>2$, the classes $\mathcal{X}_{L}$ and $\mathcal{X}_{H}$ are $L_{2}$-predictable in the weak sense uniformly with respect to the norm $\|\cdot\|_{L_{q}(\mathbf{R})}$. 
Remark 1 Since the constant $\Omega$ is the same for the classes $\mathcal{K}, \mathcal{X}_{L}, \mathcal{X}_{H}$, the set of $k(\cdot) \in \mathcal{K}$ such that the corresponding processes $y(\cdot)$ can be predicted is restricted for $x(\cdot) \in \mathcal{X}_{H}$. On the other hand, these restrictions are absent for band-limited processes $x(\cdot) \in \mathcal{X}_{L}$, since they are automatically included to all similar classes with larger $\Omega$, i.e., the constant $\Omega$ in the definition of $\mathcal{X}_{L}$ can always be increased.

The question arises how to find the predicting kernels. In the proof of Theorem 1, a possible choice of the kernels is given explicitly via Fourier transforms.

\subsection{Predictability for some bounded processes}

Let $C(\mathbf{R})$ be the Banach space of all bounded and continuous functions $f: \mathbf{R} \rightarrow \mathbf{C}$, and let $C(\mathbf{R})^{*}$ be the dual space for $C(\mathbf{R})$, i.e., it is the space of all linear continuous functionals $\xi: C(\mathbf{R}) \rightarrow \mathbf{C}$ (see, e.g., Yosida (1980)).

Let $\mathcal{M}_{\infty}$ be the class of all processes $x(t): \mathbf{R} \rightarrow \mathbf{C}$ such that there exists a function $X_{c} \in L_{1}(\mathbf{R})$, a sequence $\left\{\omega_{k}\right\}_{k=1}^{+\infty} \subset \mathbf{R}$, and a sequence $\left\{c_{k}\right\}_{k=1}^{+\infty} \subset \mathbf{C}$ such that $\sum_{k=1}^{+\infty}\left|c_{k}\right|<$ $+\infty$ and

$$
x(t)=\frac{1}{2 \pi} \sum_{k=1}^{\infty} c_{k} e^{i \omega_{k} t}+\frac{1}{2 \pi} \int_{-\infty}^{+\infty} e^{i \omega t} X_{c}(\omega) d \omega .
$$

Clearly, any set $X \triangleq\left(\left\{\omega_{k}\right\}_{k=1}^{+\infty},\left\{c_{k}\right\}_{k=1}^{+\infty}, X_{c}\right)$ with the required properties is uniquely defined by the process $x \in \mathcal{M}_{\infty}$, and can be associated with an unique element of $C(\mathbf{R})^{*}$ such that

$$
\langle f, X\rangle=\sum_{k=1}^{\infty} c_{k} f\left(\omega_{k}\right)+\int_{-\infty}^{+\infty} f(\omega) X_{c}(\omega) d \omega \quad \forall f \in C(\mathbf{R}) .
$$

In particular, $x(t)=\left\langle\frac{1}{2 \pi} e^{i t}, X\right\rangle$ for all $t$. We will denote this relationship as $X=\mathcal{F} x$, using the same notation as for the Fourier transform, and we extend Definition 3 on this case (it is a frequency representation, but not a Fourier transform anymore). As required in Definition 3, we provide the set $\{X\}$ of these sets $X$ with the norm $\|\cdot\|_{C(\mathbf{R})^{*}}$.

If $x \in \mathcal{M}_{\infty}$, then $|x(t)| \leq(2 \pi)^{-1}\left\|e^{i t} \cdot\right\|_{C(\mathbf{R})}\|X\|_{C(\mathbf{R})^{*}}$. Hence all functions from $\mathcal{M}_{\infty}$ are bounded on $\mathbf{R}$.

Let $\varepsilon \in(0, \Omega)$ be given. Let

$$
\begin{aligned}
& \mathcal{M}_{L} \triangleq\left\{x \in \mathcal{M}_{\infty}:\left|\omega_{k}\right| \leq \Omega-\varepsilon(\forall k), \quad \operatorname{supp} X_{c} \subseteq[-\Omega+\varepsilon, \Omega-\varepsilon]\right\} \\
& \mathcal{M}_{H} \triangleq\left\{x \in \mathcal{M}_{\infty}:\left|\omega_{k}\right| \geq \Omega+\varepsilon(\forall k), \quad \operatorname{supp} X_{c} \subseteq(-\infty,-\Omega-\varepsilon] \cup[\Omega+\varepsilon,+\infty)\right\} .
\end{aligned}
$$

$\mathcal{M}_{L}$ is a class of band-limited processes, and $\mathcal{M}_{H}$ is a class of high-frequency processes. 
Theorem 2 The classes $\mathcal{M}_{L}$ and $\mathcal{M}_{H}$ are $L_{\infty}$-predictable in the weak sense uniformly with respect to the norm $\|\cdot\|_{C(\mathbf{R})^{*}}$.

\section{On a model with ideal low pass-pass filter}

Corollary 1 Assume a model with a process $x(\cdot)$ such that an observer is able to decompose it as $x(t)=x_{L}(t)+x_{H}(t)$, where $x_{L}(\cdot) \in \mathcal{X}_{L} \cup \mathcal{M}_{L}$ and $x_{H}(\cdot) \in \mathcal{X}_{H} \cup \mathcal{M}_{H}$. Then this observer would be able to predict (approximately, in the sense of weak presdictability) the values of $y(t)=\int_{t}^{+\infty} k(t-s) x(s) d s$ for $k(\cdot) \in \mathcal{K}$ by predicting the processes $y_{L}(t)=$ $\int_{t}^{+\infty} k(t-s) x_{L}(s) d s$ and $y_{H}(t)=\int_{t}^{+\infty} k(t-s) x_{H}(s) d s$ separately. More precisely, the process $\widehat{y}(t) \triangleq \widehat{y}_{L}(t)+\widehat{y}_{H}(t)$ is the prediction of $y(t)$, where $y_{L}(t)=\int_{-\infty}^{t} \widehat{k}_{L}(t-s) x_{L}(s) d s$ and $y_{H}(t)=\int_{-\infty}^{t} \widehat{k}_{H}(t-s) x_{H}(s) d s$, and where $\widehat{k}_{L}(\cdot)$ and $\widehat{k}_{H}(\cdot)$ are predicting kernels which existence for the processes $x_{L}(\cdot)$ and $x_{H}(\cdot)$ is established above.

Let $\chi_{L}(\omega) \triangleq \mathbb{I}_{\{|\omega| \leq \Omega\}}$ and $\chi_{H}(\omega) \triangleq 1-\chi_{L}(\omega)=\mathbb{I}_{\{|\omega|>\Omega\}}$, where $\omega \in \mathbf{R}$.

The assumptions of Corollary 1 mean that there are a low-pass filter and a high-pass filter with the transfer functions $\chi_{L}$ and $\chi_{H}$ respectively, with $x(\cdot)$ as the input, i.e., that the values $x_{L}(s)$ and $x_{H}(s)$ for $s \leq t$ are available at time $t$, where

$x_{L}(\cdot) \triangleq \mathcal{F}^{-1} X_{L}, \quad X_{L}(\omega) \triangleq \chi_{L}(\omega) X(\omega), \quad x_{H}(\cdot) \triangleq \mathcal{F}^{-1} X_{H}, \quad X_{H}(\omega) \triangleq \chi_{H}(\omega) X(\omega)$,

and where $X \triangleq \mathcal{F} x$. It follows that the predictability in the weak sense described in Definition 3 is possible for any process $x(\cdot)$ that can be decomposed without error on a band limited process and a high-frequency process, i.e., when there is a low-pass filters which behaves as an ideal filter for this process. (Since $x_{H}(t)=x(t)-x_{L}(t)$, existence of the law pass filter implies existence of the high pass filter). On the other hand, Corollary 1 implies that the existence of ideal low-pass filters is impossible for general processes, since they cannot be predictable in the sense of Definition 3.

Clearly, processes $x(\cdot) \in \mathcal{X}_{L} \cup \mathcal{X}_{H} \cup \mathcal{M}_{L} \cup \mathcal{M}_{H}$ are automatically covered by Corollary 1, i.e., the existence of the filters is not required for this case. For instance, we have immediately that $x_{L}(\cdot)=x(\cdot)$ and $x_{H}(\cdot) \equiv 0$ for band-limited processes.

\section{Proofs}

Let $k(\cdot) \in \mathcal{K}$ and $K(i \omega)=\mathcal{F} k$. Let $(2.2)$ holds with $\delta(p)=\prod_{m=1}^{n} \delta_{m}(p)$, where $\delta_{m}(p) \triangleq$ $p-a_{m}+b_{m} i$, and where $a_{m}, b_{m} \in \mathbf{R}, p \in \mathbf{C}$. By the assumptions on $\mathcal{K}$, we have that $a_{m}>0$ and $\left|b_{m}\right|<\Omega$. 
It suffices to present a set of predicting kernels $\widehat{k}$ with desired properties. We will use a version of the construction introduced in Dokuchaev (1996) for an optimal control problem. This construction is very straightforward and does not use the advanced theory of $H^{p}$-spaces.

For $\gamma \in \mathbf{R}$, set

$$
\begin{aligned}
& \alpha_{m}=\frac{\Omega^{2}-b_{m}^{2}}{a_{m}}, \quad V_{m}(p) \triangleq 1-\exp \left(\gamma \frac{p-a_{m}+b_{m} i}{p+\alpha_{m}-b_{m} i}\right), \quad V(p) \triangleq \prod_{m=1}^{n} V_{m}(p), \\
& \widehat{K}(i \omega) \triangleq V(i \omega) K(i \omega) .
\end{aligned}
$$

Lemma $1 \quad$ (i) $V(p) \in H^{\infty}$ and $\widehat{K}(p) \triangleq K(p) V(p) \in H^{2} \cap H^{\infty}$;

(ii) If $\gamma>0$ and $\omega \in[-\Omega, \Omega]$, then $|V(i \omega)| \leq 2$. If $\gamma<0$, and if $\omega \in \mathbf{R},|\omega| \geq \Omega$, then $|V(i \omega)| \leq 2$.

(iii) If $\omega \in(-\Omega, \Omega)$, then $V(i \omega) \rightarrow 1$ as $\gamma \rightarrow+\infty$. If $\omega \in \mathbf{R}$ and $|\omega|>\Omega$, then $V(i \omega) \rightarrow 1$ as $\gamma \rightarrow-\infty$.

(iv) For any $\varepsilon>0, V(i \omega) \rightarrow 1$ as $\gamma \rightarrow+\infty$ uniformly in $\omega \in[-\Omega+\varepsilon, \Omega-\varepsilon]$ as $\gamma \rightarrow+\infty$, and $V(i \omega) \rightarrow 1$ as $\gamma \rightarrow-\infty$ uniformly in $\omega \in \mathbf{R}$ such that $|\omega| \geq \Omega+\varepsilon$.

Proof of Lemma 1. Clearly, $V_{m}(p) \in H^{\infty}$, and $\delta_{m}(p)^{-1} V_{m}(p) \in H^{2} \cap H^{\infty}$, since the pole of $\delta_{m}(p)^{-1}$ is being compensated by multiplying on $V_{m}(p)$. It follows that $K(p) V(p) \in$ $H^{2} \cap H^{\infty}$. Then statement (i) follows.

Further, for $\omega \in \mathbf{R}$,

$$
\begin{aligned}
& \frac{i \omega-a_{m}+b_{m} i}{i \omega+\alpha_{m}-b_{m} i}=\frac{\left(-a_{m}+i \omega+i b_{m}\right)\left(\alpha_{m}-i \omega+b_{m} i\right)}{\left(\omega-b_{m}\right)^{2}+\alpha_{m}^{2}} \\
& =\frac{-a_{m} \alpha_{m}+\left(\omega+b_{m}\right)\left(\omega-b_{m}\right)}{\left(\omega-b_{m}\right)^{2}+\alpha_{m}^{2}}+i \frac{-a_{m}\left(\omega+b_{m}\right)+\alpha_{m}\left(\omega+b_{m}\right)}{\left(\omega-b_{m}\right)^{2}+\alpha_{m}^{2}} .
\end{aligned}
$$

Then

$$
\operatorname{Re} \frac{i \omega-a_{m}+b_{m} i}{i \omega+\alpha_{m}-b_{m} i}=\frac{-a_{m} \alpha_{m}+\omega^{2}-b_{m}^{2}}{\left(\omega-b_{m}\right)^{2}+\alpha_{m}^{2}}=\frac{\omega^{2}-\Omega^{2}}{\left(\omega-b_{m}\right)^{2}+\alpha_{m}^{2}} .
$$

Then statements (ii)-(iv) follow. This completes the proof of Lemma 1.

Proof of Theorem 1. For $x(\cdot) \in L_{2}(\mathbf{R})$, let $X \triangleq \mathcal{F} x, Y \triangleq \mathcal{F} y=K(i \omega) X(\omega)$. Let $V$ be as defined above. Set $\widehat{Y}(\omega) \triangleq \widehat{K}(i \omega) X(\omega)=V(i \omega) Y(\omega)$.

Let us consider the cases of $\mathcal{X}_{L}$ and $\mathcal{X}_{H}$ simultaneously. For the case of the class $\mathcal{X}_{L}$, consider $\gamma>0$ and assume that $\gamma>0$ and $\gamma \rightarrow+\infty$. Set $D \triangleq[-\Omega, \Omega]$ for this case. For the case of the class $\mathcal{X}_{H}$, consider $\gamma<0$ and assume that $\gamma<0$ and $\gamma \rightarrow-\infty$. Set $D \triangleq(-\infty,-\Omega] \cup[\Omega,+\infty)$ for this case. 
Let $x(\cdot) \in \mathcal{X}_{L}$ or $x(\cdot) \in \mathcal{X}_{H}$. In both cases, Lemma 1 gives that $|V(i \omega)| \leq 1$ for all $\omega \in D$. If $\gamma \rightarrow+\infty$ or $\gamma \rightarrow-\infty$ respectively for $\mathcal{X}_{L}$ or $\mathcal{X}_{H}$ cases, then $V(i \omega) \rightarrow 1$ for a.e. $\omega \in D$, i.e., for a.e. $\omega$ such that $X(\omega) \neq 0$.

Let us prove (i). Since $K(i \omega) \in L_{\infty}(\mathbf{R})$ and $X \in L_{2}(\mathbf{R})$, we have that $Y(\omega)=$ $K(i \omega) X(\omega) \in L_{2}(\mathbf{R})$ and $\widehat{Y} \in L_{2}(\mathbf{R})$. By Lemma 1, it follows that

$$
\widehat{Y}(\omega) \rightarrow Y(\omega) \text { for a.e. } \omega \in \mathbf{R},
$$

as $\gamma \rightarrow+\infty$ or $\gamma \rightarrow-\infty$ respectively for $\mathcal{X}_{L}$ or $\mathcal{X}_{H}$ cases. We have that $X \in L_{2}(\mathbf{R})$, $K(i \omega) \in L_{2}(\mathbf{R}) \cap L_{\infty}(\mathbf{R})$ and

$$
\begin{aligned}
& |\widehat{K}(i \omega)-K(i \omega)| \leq|V(i \omega)-1||K(i \omega)| \leq 2|K(i \omega)|, \quad \omega \in D, \\
& |\widehat{Y}(\omega)-Y(\omega)| \leq 2|Y(\omega)|=2|K(i \omega)||X(\omega)|, \quad \omega \in D .
\end{aligned}
$$

By (5.1),(5.3), and by Lebesque Dominance Theorem, it follows that

$$
\|\widehat{Y}-Y\|_{L_{2}(\mathbf{R})} \rightarrow 0, \quad \text { i.e., } \quad\|\widehat{y}-y\|_{L_{2}(\mathbf{R})} \rightarrow 0
$$

as $\gamma \rightarrow+\infty$ or $\gamma \rightarrow-\infty$ respectively for $\mathcal{X}_{L}$ or $\mathcal{X}_{H}$ cases, where $\widehat{y}=\mathcal{F}^{-1} \widehat{Y}$.

Let us prove (ii)-(iii). Take $d=1$ for (ii) and take $d=2$ for (iii). If $X \in L_{q}(\mathbf{R})$ for $q>d$, then Hölder inequality gives

$$
\|\widehat{Y}-Y\|_{L_{d}(\mathbf{R})} \leq\|\widehat{K}(i \omega)-K(i \omega)\|_{L_{\mu}(D)}\|X\|_{L_{q}(D)}
$$

where $\mu$ is such that $1 / \mu+1 / q=1 / d$. By (5.2) and by Lebesque Dominance Theorem again, it follows that

$$
\|\widehat{K}(i \omega)-K(i \omega)\|_{L_{\mu}(D)} \rightarrow 0 \quad \forall \mu \in[1,+\infty)
$$

as $\gamma \rightarrow+\infty$ or $\gamma \rightarrow-\infty$ respectively for $\mathcal{X}_{L}$ or $\mathcal{X}_{H}$ cases. By (5.5)-(5.6), it follows that the predicting kernels $\widehat{k}(\cdot)=\widehat{k}(\cdot, \gamma)=\mathcal{F}^{-1} \widehat{K}(i \omega)$ are such as required in statements (ii)-(iii). This completes the proof of Theorem 1 .

Proof of Theorem 2. For $x(\cdot) \in \mathcal{M}_{\infty}$ such that $X=\left(\left\{\omega_{k}\right\}_{k=1}^{+\infty},\left\{c_{k}\right\}_{k=1}^{+\infty}, X_{c}\right)$, we have that the corresponding set $Y=\mathcal{F} y$ is $Y=\left(\left\{\omega_{k}\right\}_{k=1}^{+\infty},\left\{K\left(i \omega_{k}\right) c_{k}\right\}_{k=1}^{+\infty}, K(i \omega) X_{c}(\omega)\right)$. Similarly to $X$, it can be considered as an element of $C(\mathbf{R})^{*}$ such that $y(t)=\left\langle\frac{1}{2 \pi} e^{i t}, Y\right\rangle$. Let $V$ and $\widehat{K}$ be as defined above. Set

$$
\widehat{Y} \triangleq\left(\left\{\omega_{k}\right\}_{k=1}^{+\infty},\left\{\widehat{K}\left(i \omega_{k}\right) c_{k}\right\}_{k=1}^{+\infty}, \widehat{K}(i \omega) X_{c}(\omega)\right)
$$

It can be seen as an element of $C(R)^{*}$, and $\widehat{y}(t)=\int_{-\infty}^{t} \widehat{k}(t-s) x(s) d s=\left\langle\frac{1}{2 \pi} e^{i t}, \widehat{Y}\right\rangle$, where the kernel is defined via inverse Fourier transform $\widehat{k}(\cdot)=\mathcal{F}^{-1} \widehat{K}(i \omega)$. 
We consider the cases of $\mathcal{M}_{L}$ and $\mathcal{M}_{H}$ simultaneously. For the case of the class $\mathcal{M}_{L}$, we consider $\gamma>0$ and $\gamma \rightarrow+\infty$. Set $D_{\varepsilon} \triangleq[-\Omega+\varepsilon, \Omega-\varepsilon]$ for this case. For the case of the class $\mathcal{M}_{H}$, we consider $\gamma<0$ and $\gamma \rightarrow-\infty$. Set $D_{\varepsilon} \triangleq(-\infty,-\Omega-\varepsilon] \cup[\Omega+\varepsilon,+\infty)$ for this case.

Let $x(\cdot) \in \mathcal{M}_{L}$ or $x(\cdot) \in \mathcal{M}_{H}$. In both cases, Lemma 1 gives that $|V(i \omega)| \leq 2$ for all $\omega \in D_{\varepsilon}$. If $\gamma \rightarrow+\infty$ or $\gamma \rightarrow-\infty$ respectively for $\mathcal{M}_{L}$ or $\mathcal{M}_{H}$ cases, then $V(i \omega) \rightarrow 1$ uniformly in $\omega \in D_{\varepsilon}$. Hence $\|\widehat{K}-K\|_{L_{\infty}\left(D_{\varepsilon}\right)} \rightarrow 0$ as $\gamma \rightarrow+\infty$ or $\gamma \rightarrow-\infty$, for the cases of $\mathcal{M}_{L}$ and $\mathcal{M}_{H}$, respectively. If $x \in \mathcal{M}_{L}$ or $x \in \mathcal{M}_{H}$, then

$$
|\langle f, X\rangle| \leq \max _{t \in D_{\varepsilon}}|f(t)|\|X\|_{C(\mathbf{R})^{*}} \quad \forall f \in C(\mathbf{R}), \quad X=\mathcal{F} x
$$

Hence

$|\widehat{y}(t)-y(t)|=\left|\left\langle\frac{1}{2 \pi} e^{i t \cdot}, \widehat{Y}-Y\right\rangle\right|=\left|\left\langle\frac{1}{2 \pi} e^{i t \cdot}(\widehat{K}-K), X\right\rangle\right| \leq \frac{1}{2 \pi}\|\widehat{K}-K\|_{L_{\infty}\left(D_{\varepsilon}\right)}\|X\|_{C(\mathbf{R})^{*}}$

for all $t \in \mathbf{R}$. Then the proof of Theorem 2 follows.

Corollary 1 follows immediately from Theorem 1.

Remark 2 Formally, the corresponding predictors require the past values of $x(s)$ for all $s \in(-\infty, t]$, but it is not too restrictive, since $\int_{-\infty}^{t} \widehat{k}(t-s) x(s) d s$ can be approximated by $\int_{-M}^{t} \widehat{k}(t-s) x(s) d s$ for large enough $M>0$. In addition, the corresponding transfer functions can be approximated by rational fraction polynomials, and more general kernels $k$ can be approximated by kernels from $\mathcal{K}$.

Remark 3 The system for the suggested predictors is stable, since the corresponding transfer functions have poles in the domain $\{\operatorname{Re} z<0\}$ only. However, the suggested predictors are not robust. For instance, if the predictor is designed for the class $\mathcal{X}_{L}$ and it is applied for a process $x(\cdot) \notin \mathcal{X}_{L}$ with small non-zero energy at the frequencies outside $[-\Omega, \Omega]$, then the error generated by the presence of this energy is increasing if $\gamma \rightarrow \infty$.

\section{References}

[1] Beutler F.G. (1966). Error-free recovery of signals from irregularly spaced samples. SIAM Review, 8(3), 328-335.

[2] Brown J.R., Jr.. (1969). Bounds for truncation error in sampling expansion of bandlimited signals. IEEE Transactions Inform. Theory 15, no. 4, 440-444.

[3] Dokuchaev N.G. (1996). Suboptimal damping of forced oscillations. Vestnik St. Petersburg University: Mathematics. 29, Iss. 4, 49-51. 
[4] Duren P. (1970) Theory of $H^{p}$-Spaces. Academic Press, New York.

[5] Knab J.J. (1979). Interpolation of band-limited functions using the approximate prolate series. IEEE Transactions on Information Theory 25(6), 717-720.

[6] Lyman R.J, Edmonson W.W., McCullough S., and Rao M. (2000). The predictability of continuous-time, bandlimited processes. IEEE Transactions on Signal Processing 48, Iss. 2, 311-316.

[7] Lyman R.J and Edmonson W.W. (2001). Linear prediction of bandlimited processes with flat spectral densities. IEEE Transactions on Signal Processing 49, Iss. 7, 15641569 .

[8] Marvasti F. (1986). Comments on "A note on the predictability of band-limited processes." Proceedings of the IEEE, 74(11), 1596.

[9] Papoulis A. (1985). A note on the predictability of band-limited processes. Proceedings of the IEEE, $\mathbf{7 3}(8), 1332-1333$.

[10] Slepian D. (1978). Prolate spheroidal wave functions, Fourier analysis, and uncertainty-V: The discrete case. Bell System Technical Journal, 57(5), 1371-1430.

[11] Vaidyanathan P.P. (1987). On predicting a band-limited signal based on past sample values. Proceedings of the IEEE, 75(8), 1125-1127.

[12] Wainstein L.A. and Zubakov V.D. (1962). Extraction of Signals from Noise. Englewood Cliffs, NJ: Prentice-Hall.

[13] Yosida K. (1980). Functional Analysis. Springer-Verlag, N.Y. 